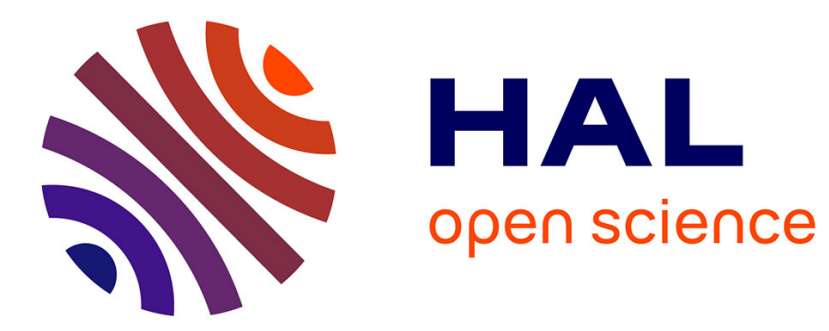

\title{
L'ACIDE PANTOTHÉNIQUE DANS LES PRODUITS LAITIERS
}

\author{
A. Houdinière
}

\section{To cite this version:}

A. Houdinière. L'ACIDE PANTOTHÉNIQUE DANS LES PRODUITS LAITIERS. Le Lait, 1950, 30 (291_292), pp.37-40. hal-00928007

\section{HAL Id: hal-00928007 https://hal.science/hal-00928007}

Submitted on 1 Jan 1950

HAL is a multi-disciplinary open access archive for the deposit and dissemination of scientific research documents, whether they are published or not. The documents may come from teaching and research institutions in France or abroad, or from public or private research centers.
L'archive ouverte pluridisciplinaire HAL, est destinée au dépôt et à la diffusion de documents scientifiques de niveau recherche, publiés ou non, émanant des établissements d'enseignement et de recherche français ou étrangers, des laboratoires publics ou privés. 
(Travail du Laboratoire de Physiologie de la Nutrition de l'Ecole des Hautes Etudes et du Centre de Recherches sur l'Alimentation. rationnelle de l'Institut National de la Recherche Agronomique.)

\title{
BIBLIOGRAPHIE
}

[1] R, C. Bender et G. C. Supplee. Amer. Journ. Dis. Child., 1933, 45, 995.

[2] F. C. Sohomeneber, H. Steenbock et C. A. Bauman. Wisc. Agrio. Exp. - Station Bull., 1936, 435, 66.

[3] Callon et Hixon, eités par G. Genin. Le Lait, 1937, 17, 368.

[4] L. Randoin et H. Simonnet. Les vitamines, 4e édit., A. Colin, édit., Paris, 1942, p. 90.

[5] J. Botsselot et J. Causeret. Bull. Soe. Se. Hyg. alim., 1946, 34, 148. (Le Lait, 1948, 28, 26.)

[6] J. Boisgelot et J. Causeret. Bull. Soc. Sc. Hyg. alim., 1946, 34, 176. (Le Lait, 1948, 28, 145.)

\section{REVUE}

\section{L'ACIDE PANTOTHÉNIQUE DANS LES PRODUITS LAITIERS}

\author{
par
}

\section{A. HOUDINIÊRE}

Nouvelle vitamine du groupe B, l'acide pantothénique a fait l'objet, en ces dernières années, de nombreuses recherches.

Dans un certain nombre de notes bibliographiques, avec indications de références, J. MaILlaAR et J. CAUSERet ont fait le point de nos connaissances sur cet acide. Les données suivantes, tirées de cette étude, et touchant le domaine du lait, sont susceptibles de retenir l'attention du lecteur.

L'acide pantothénique joue un rôle capital dans la nutrition des organismes supérieurs. Il est présent dans un grand nombre d'extraits de tissus animaux et végétaux. On a pu mettre en évidence ses principales propriétés et l'isoler à l'état sensiblement pur.

Sa formule développée est la suivante :

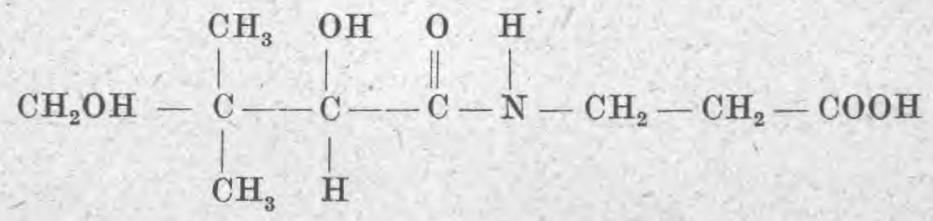

(1) Bull. Soc. Sc. Hygiène alimentaire, t. XXV, 1947, 58-60-61-91-152-155-157. 
Le tableau suivant indique les résultats du dosage de cet acide dans le lait et quelques-uns de ses dérivés.

Milligr. pour 100 grammes

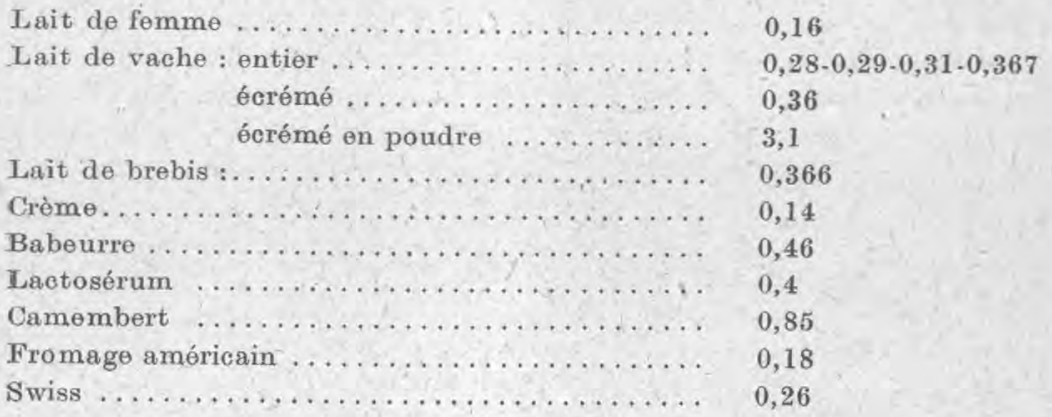

Les produits laitiers sont en général peu riches en acide pantothénique par opposition aux organes des animaux comme le foie (de 5,4 à 7,6 ), au jaune d'ouf $(6,3)$ et à la levure sèche $(4,2)$.

D'une façon générale on peut dire que l'acide pantothénique est une vitamine de croissance qui conditionne la multiplication 'des cellules. Il intervient dans la nutrition des revêtements cutanés, des tissus nerveux et dans la physiologie de la digestion.

Son rôle vitaminique a été mis en évidence chez le poulet, la souris, le chien, le porc et l'homme.

Sa carence chez le poulet détermine une dermatose atteignant principalement les yeux, les coins de la bouche et les pattes.

Chez le rat on observe un arrêt de croissance, un raidissement et'un grisonnement des poils noirs (achromotrichie), une dermatose et une excrétion de porphyrine.

Le pore carencé présente des troubles de la croissance pondérale (chute de poids et ralentissement de croissance), des troubles cutanés (rudesse et chuté des poils), des troubles digestifs (diarrhés, graves inflammations du colon) et des troubles nerveux (démarches anormales avec chutes soudaines, dégénérescence des racines postérieures des nerfs rachidiens et de leurs ganglions).

Chez l'homme, l'acide pantothénique semble intervenir dans l'évolution des anémies jusqu'à présent traitées par l'hépatothérapie. L'anémie d'Addison-Bermer aurait des syndromes communsavec l'avitaminose : troubles gastro-intestinaux, dégénérescence graisseuse du foie, lésions de la moelle épinière chez le poulet carencé et dégénérescence des fibres longues des cordons postérieurs chez les hommes atteints d'anémie. L'acide pantothénique jouerait un rôle dans la formation des globules rouges. Ce problème n'est 
toutefois pas entièrement résolu et demande de nouvelles recherches.

L'acide pantothénique est dosé par voie microbiologique ou par voie biologique. Les méthodes chimiques n'ont pas encore donné de résultats satisfaisants.

Dans le premier cas, on utilise le rôle vitaminique dé cet acide à l'égard des bactéries lactiques. La méthode de dosage consiste à mesurer la vitesse de croissance de ces microbes (Lactobacillus Casei, Helveticus, Arabinosus, Bulgaricus, ou Streptococcus lactis, etc.).

On ne peut employer cette méthode qu'après avoir extrait la vitamine des tissus par solution aqueuse à l'autoclave, à l'autolyse ou à l'hydrolyse enzymatique. L'acide pantothénique est en effet instable aux acides et aux alcalis.

La méthode biologique fait appel le plus souvent aux propriétés antidermatiques de l'acide chez le poulet.

On eonnaît de nombreuses anti-vitamines de l'acide pantothénique; leur structure chimique est tantôt très voisine de la sienne (acide sulfopantothénique, etc.) tantôt très éloignée (sulfanilamide). Cette question comporte encore de nombreuses inconnues.

La carence du porc, qui entraîne des troubles graves, a conduit les chercheurs à préciser les besoins de cet animal en acide pantothénique. Les besoins minima seraient compris entre $0 \mathrm{mgr} .17$ et 0 mgr. 26 par kilogramme de poids vif et par jour. Une dose de 0 migr. 51 protège le porc de la dégénérescence nerveuse.

Le Comité de nutrition animal du National Research Council recommande la dose de 0 mgr. 41 calculée d'après la quantité d'acide pantothénique que renferme la ration alimentaire normale du pore.

\section{BIBLIOGRAPHIE}

R. J. Williams et eoll. Journ. of Amer. Chem. Soe., 1931, 53, 738; $1931,53,4171$.

R. J. Williams et coll. Journ, of Amer. Chem. Soc., 1932, 54, 3462; $1933,55,2912$.

R. J. Williams et R. Moser. Journ. of Amer. Chem. Soe., 1934, 56, 169.

R. J. Willrams et coll. Journ. of Amer. Chem. Soc., 1938, 60, 2719; $1939,61,454 ; 1939,61,1421 ; 1940,62,1776$.

R. J. Williams et R. T. MajoR. Science, 1940, 91, 245.

E. T. Strluer et coll. Journ. of Amer. Schem. Soc., 1940, 62, 1779.

Williams et coll. The Univ. Texas Publ, n n $^{\circ} 4237,1942,97$.

T. H. Jukks. Journ, of Nutr., 1941, 21, 193.

V. H. Cheldelin et R. J. Wilutams. The Univ. Texas Publ, no 4237 , $1942,105$.

A. Z. HoDson. Journ. of Nutr., 1945, 29, 137.

P. B. Pearson et A. L. Darnell. Journ. of Nutr, 1946, 31, 51.

J. C. Bauernfeind et coll. Poultry Sc, 1942, 21, 136.

H. A. WatsMan et coll. Journ. of Nutr., 1942, 23, 239. 
T. H. JuKes. Journ. of Amer. Chem. Soe., 1939, 61, 975; Journ, of Biol. Chem., 1939, 129, 225.

J. G. SAndza ot L. R. Cerécedo, Journ. of Nutr., 1941, 21, 609.

J. M. KrBbin et coll. Amer. Journ, of Physio., 1939, 128, 102.

E. H. Hugues. Journ. of A gr. Res., 1942, 64, 185.

T. D. Spies et coll. Journ. of Amer. Med. Assos., 1940, 115, 523.

S. Lepkovsky et T. H, JukEs. Journ. of Biol. Chem., 1935, 111, 119 ; $1936,114,109$.

T. H. JuкEs. Journ. of Biol. Chem., 1937, 117, 11; 1939, 129, 225.

M. K. Diмick et A. Lepp. Journ. of Nutr., 1940, 20, 413.

B. LYтнсов et coll. Bioch. Journ., 1940, 34, 1335.

H. D. WEST et coll, Journ. of Nutr, 1943, 25, 471.

M. M. Wintrobe et coll. Journ. of Nutr., 1942, 24, 345; Bull. Johns Hopkins Hosp., 1943, 73, 313.

M. Piette. Bull. Soc. Ohim. Biol., 1946, 28, 322.

F UлітA et AлIsAка. Zitschr. für Biochem., 1941, 308, 430.

G. LUNDE. Skand. Vet., 1940, 30, 26.

\section{BIBLIOGRAPHIE ANALYTIQUE}

\section{LES LIVRES}

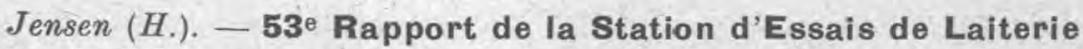
de I'Etat danois. 1 brochure de 50 pages. Prix : 2 cour. dan. En vente chez : August Bang, 60, Vesterbrogade, Copenhague V.

I. Essai du pasteurisateur à plaques Kolding avec chauffage à eau, type 2 SK.

Cet appareil à plaques, construit par "De Danske Mejeriers Maskinfabrik n à Kolding (Danemark) sert à la pasteurisation du lait entier par pasteurisation haute ou basse et est chauffé à l'eau chaude.

La température de pasteurisation ne doit pas être inférieure à $84^{\circ}$ si on veut que le lait donne un résultat négatif à l'épreuve de Storeh pendant 3 jours. Le lait pasteurisé par pasteurisation haute est satisfaisant au point de vue bactériologique. $18,5 \%$ de l'albumine sont précipités par le chauffage à $85^{\circ}$ et $27,2 \%$ par lo chauffage à $90^{\circ}$. Le lait traité par pasteurisation basse $\left(72^{\circ}\right)$ a développé un maximum de 2,5 unités bleues lors de l'épreuve de la phosphatase de Scharer (durée de l'ineubation : 3 heures). La pasteurisation basse a réduit de façon satisfaisante la teneur du lait en bactéries. On n'a pas trouvé de colibacilles dans le lait chauffé à au moins 66\%. La précipitation d'albumine soluble est minime : $5 \%$ à $72-73^{\circ}$. La faculté de eoagulation du lait n'est réduite que de $0,6 \%$ à $72^{\circ}$ et de $1,3 \%$ à $73^{\circ}$. Le chauffage du lait à $73^{\circ}$ n'a pas produit de réduction de la montée de la crème par rapport au lait cru, les échantillons étant conservés à $10^{\circ}$. Lorsque les échantillons furent conservés au froid, la montée de la crème du lait ehauffé fut un peu moindre que celle du lait cru. La pasteurisation basse a considérablement augmenté la faculté de conservation du lait. Les plaques et la cellule de chambrage de l'appareil sont d'une excellente construction au point 